\title{
Spin and Valley States in Gate-Defined Bilayer Graphene Quantum Dots
}

\author{
Marius Eich, ${ }^{1, *}$ František Herman, ${ }^{2}$ Riccardo Pisoni,${ }^{1}$ Hiske Overweg, ${ }^{1}$ Annika Kurzmann, ${ }^{1}$ Yongjin Lee, ${ }^{1}$ \\ Peter Rickhaus, ${ }^{1}$ Kenji Watanabe, ${ }^{3}$ Takashi Taniguchi, ${ }^{3}$ Manfred Sigrist, ${ }^{2}$ Thomas Ihn, ${ }^{1}$ and Klaus Ensslin ${ }^{1}$ \\ ${ }^{1}$ Solid State Physics Laboratory, ETH Zurich, 8093 Zurich, Switzerland \\ ${ }^{2}$ Institute for Theoretical Physics, ETH Zurich, 8093 Zurich, Switzerland \\ ${ }^{3}$ Advanced Materials Laboratory, NIMS, 1-1 Namiki, Tsukuba 305-0044, Japan
}

(Received 28 March 2018; revised manuscript received 7 June 2018; published 24 July 2018; corrected 8 December 2020)

\begin{abstract}
In bilayer graphene, electrostatic confinement can be realized by a suitable design of top and back gate electrodes. We measure electronic transport through a bilayer graphene quantum dot, which is laterally confined by gapped regions and connected to the leads via $p$ - $n$ junctions. Single electron and hole occupancy is realized and charge carriers $n=1,2, \ldots 50$ can be filled successively into the quantum system with charging energies exceeding $10 \mathrm{meV}$. For the lowest quantum states, we can clearly observe valley and Zeeman splittings with a spin $g$-factor of $g_{s} \approx 2$. In the low-field limit, the valley splitting depends linearly on the perpendicular magnetic field and is in qualitative agreement with calculations.
\end{abstract}

DOI: 10.1103/PhysRevX.8.031023

Subject Areas: Condensed Matter Physics,

Quantum Physics,

Semiconductor Physics

\section{INTRODUCTION}

Graphene has been recognized early on as a prime candidate to host spin qubits [1]. With carbon being one of the lightest elements in the periodic table, spin-orbit interactions are expected to be weak. In addition, $99 \%$ of natural carbon consists of nuclear spin-free ${ }^{12} \mathrm{C}$. Therefore, the two main spin decoherence mechanisms for spin qubits, namely, spin-orbit interactions and hyperfine coupling of nuclear and electronic spins, should be strongly suppressed in any carbon-based solid state system. So far, these theoretical considerations have not come to fruition in experiments.

Until now, graphene quantum dots (QDs) have been mostly realized by top-down lithography and etching of single layer graphene [2-6]. While many of the basic quantum transport properties such as Coulomb blockade [2,3], charge detection [7], and electronic phase coherence $[8,9]$ have been experimentally demonstrated, the understanding of the orbital and spin character of specific states has remained elusive. In retrospect, we understand that the Coulomb blockade in these devices arises mostly from localized states at the sample edges, which remain rough on

\footnotetext{
*meich@phys.ethz.ch
}

Published by the American Physical Society under the terms of the Creative Commons Attribution 4.0 International license. Further distribution of this work must maintain attribution to the author(s) and the published article's title, journal citation, and DOI. the atomic scale because of the limitations of top-down technology [6].

More than a decade ago, experiments showed that a band gap can be opened in bilayer graphene by vertical electric fields [10-12], and charge carrier confinement in bilayer graphene has been studied in theory [13-15]. Several attempts to use split-gate electrodes to laterally confine charge carriers in the absence of a magnetic field have suffered from limited resistance values that can be experimentally obtained upon pinch-off and did not reach the last-electron regime [16-18]. Recently, we realized quantum point contacts that display quantized conductance and show pinch-off resistances orders of magnitude larger than the quantum of resistance $h / e^{2}$ [19], a necessary requirement to electrically isolate charge carriers from their environment. Here, the same fabrication technique has been adapted using suitable gate geometries to prepare QDs with an electronic quality that matches what has been achieved in the traditional semiconductors $\mathrm{Si}$ and GaAs [20,21].

In the first experiment, we demonstrate charging of a bilayer graphene QD with a single and a few holes when coupling the QD to $n$-type source and drain leads through $p-n$ tunnel barriers. Charging energies in excess of $10 \mathrm{meV}$ are observed. We reverse the gate voltages and investigate single or few electron QDs connected to $p$-type leads, demonstrating the ambipolar operation of these QDs on the same graphene flake in close vicinity to each other. Applying perpendicular magnetic fields in the second experiment, we extract the single particle level spectrum, showing 
shell filling and orbital degeneracy. The pronounced valley splitting is in agreement with calculations, which predict that the splitting depends on the dot size. In the third experiment, we carefully align the graphene sheet hosting the QD to an inplane magnetic field and find a Zeeman splitting with a $g$-factor $g_{s}=2.08 \pm 0.22$, agreeing with the expected value for carbon-based devices [22,23].

Our bilayer QDs display high-quality electronic properties comparable to standard semiconductor structures that have been optimized for the last 30 years. While excellent devices have also been reported for carbon nanotube QDs [24-28], graphene offers the advantage of a planar technology [21] and the possible combination with other 2D materials [29-31]. Our demonstration of excellent control and reproducibility opens up a wide field of possibilities for carbon-based quantum electronics.

\section{CHARACTERIZATION}

We investigated the bilayer graphene device encapsulated in hexagonal boron nitride [32-34] shown in Fig. 1. The individual layers of the van der Waals heterostructure were stacked and processed as in Ref. [19], protecting the natural edges of the bilayer flake [white dashed lines in Fig. 1(a)]. Opposite voltages applied to the split gates (green in Fig. 1) and the graphite back gate [red solid lines in Fig. 1(a)] lead to the formation of a band gap in the bilayer regions underneath the split gates. For appropriate voltages applied to theses gates, the Fermi level is tuned to be in the band gap (for details, see Ref. [19]), rendering these regions insulating and defining approximately 100-nm-wide channels between the source and drain contacts (contacts shown in yellow in Fig. 1). Finger gates [blue in Fig. 1, numbered 1 through 11 in the $x$-direction; see Fig. 1(a)] crossing the channel on top of the two split gate pairs (insulated from them by $25 \mathrm{~nm}$ of $\mathrm{Al}_{2} \mathrm{O}_{3}$ ) are biased to control the charge carrier density locally in the channel.

First, we investigate the conductance of the device, biasing only the uniform top gate crossing the entire width of the bilayer region [white asterisk in Fig. 1(a)]. By applying large opposite voltages to the graphite back gate and this top gate, the strong displacement field opens a band gap in the bilayer region underneath the top gate. The two-terminal resistance measured between the source and drain contacts reaches values on the order of $\mathrm{G} \Omega$ when tuning the Fermi level into the gap (see Appendix B), demonstrating the high electronic quality of our sample and the excellent insulating behavior of the gapped region. Biasing either pair of split gates in this regime of high displacement field, charge carriers are laterally confined and forced to flow through the narrow channel between the split gates. This is the regime in which we form and operate our QDs.

\section{RESULTS}

\section{A. Gate-defined quantum dots}

In the first experiment at $1.7 \mathrm{~K}$, we investigate charging a QD with single holes. We measured nine QDs in total, all showing qualitatively the same behavior. By recording conductance maps as a function of finger gate and split gate voltage, a particular QD can be tuned to an optimal operation point (see Appendix B). Figure 2(a) shows the conductance of the device as a function of the finger gate voltage $V_{\mathrm{FG}}$. Charge carriers can only flow through the narrow channel, because the regions underneath the split gates are insulating. The positive back gate voltage $V_{\mathrm{BG}}$ induces a finite excess electron density in the channel. With decreasing finger gate voltage $V_{\mathrm{FG}}$, the electron density is
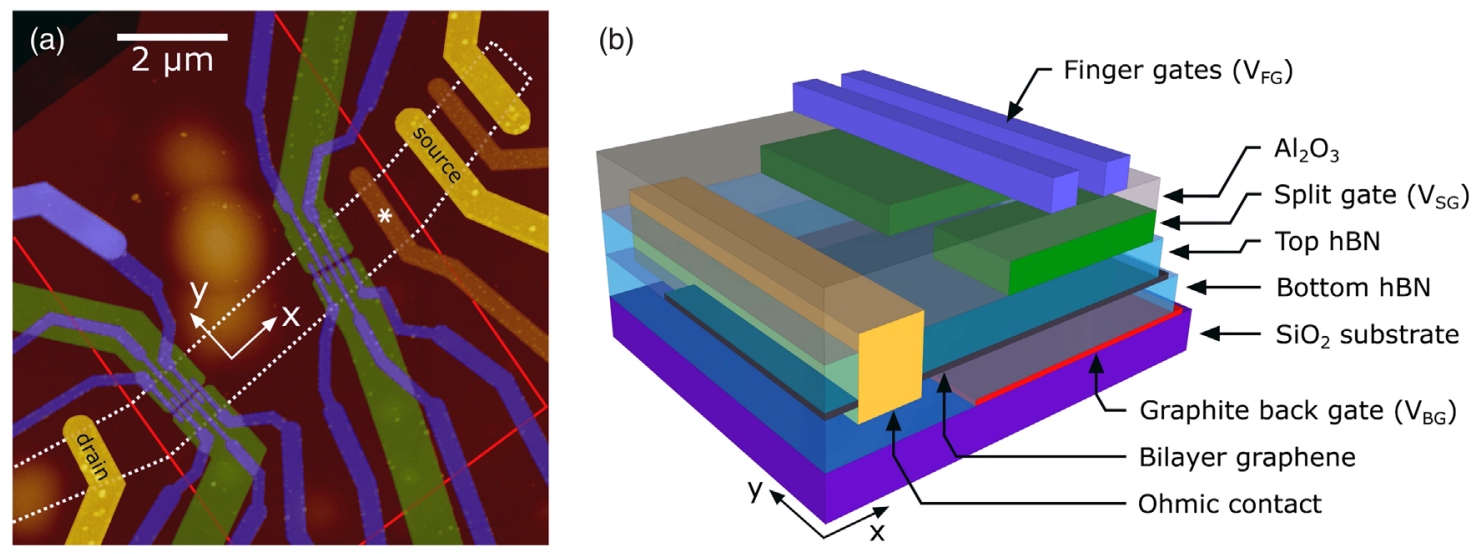

FIG. 1. (a) False color scanning force micrograph of the device. (b) 3D sketch of part of the device showing the different layers of gates and dielectrics. White dashed lines and solid red lines in (a) outline the bilayer flake and graphite back gate, respectively. Edge contacts to the bilayer are colored in yellow, split gates are shown in green, and the finger gates are shown in blue. The top gate spanning the entire width of the bilayer flake is marked with an asterisk. 

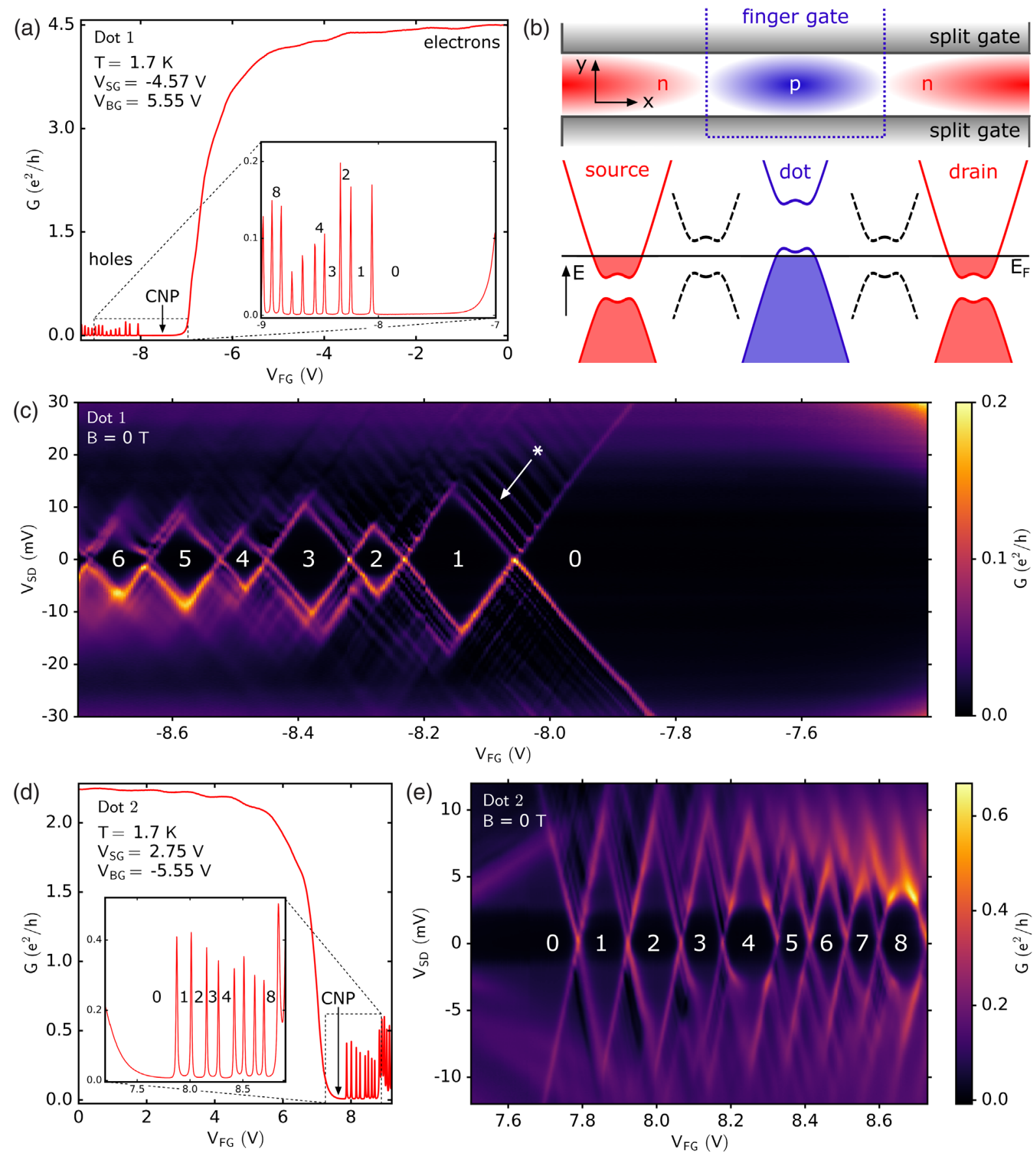

FIG. 2. (a) Conductance trace for $p$-type QD 1. (b) Schematic of the band structure at different positions along the current direction in the channel. Black dashed lines schematically show the band alignment between the dot region and the leads. (c) Coulomb diamonds in the hole regime for QD 1, with an asterisk indicating regularly spaced resonances parallel to the diamond boundaries. (d) Conductance trace and (e) Coulomb diamonds for the $n$-type QD 2. Numbers in (a) and (c), as well as (d) and (e) indicate the occupation of the QDs with holes or electrons, respectively.

locally reduced until complete pinch-off is reached at the charge neutrality point (CNP) at about $-7.5 \mathrm{~V}$.

For $V_{\mathrm{FG}}<-7.5 \mathrm{~V}$, the region underneath the finger gate is tuned into the hole regime as shown schematically in Fig. 2(b): We sketch at the top the $n$-type channel (red) with the locally induced $p$-type region (blue). Below, we show the dispersion relation near the $K$-point of the first Brillouin zone in the three spatial regions and at the $p$ - $n$ junctions between them (dashed). At the $p-n$ junctions, the Fermi level $E_{F}$ lies in the gap leading to a region with zero charge carrier density. These regions provide natural tunnel barriers separating the p-type dot from the n-type leads. By lowering the finger gate voltage in this regime, the p-type QD can be charged one by one with individual holes. 
This is seen in Fig. 2(a) at $V_{\mathrm{FG}}<-7.5 \mathrm{~V}$, where sharp conductance resonances appear (see also inset).

Finite DC bias spectroscopy of the QD tuned to this regime yields the Coulomb diamonds shown in Fig. 2(c). For $V_{\mathrm{FG}}>-8 \mathrm{~V}$, we do not see additional states contributing to transport through the QD, indicating a completely depleted dot. Therefore, we label each diamond with the occupation number of the QD [cf. inset of Fig. 2(a)]. The regularly spaced features running parallel to the edge of the Coulomb diamonds [indicated by an asterisk in Fig. 2(c)] appear for all measured QDs, are stable over a temperature range from $50 \mathrm{mK}$ to $1.7 \mathrm{~K}$, and are currently still under investigation.

To form an electron QD connected to $p$-type leads, we reverse all applied gate voltages with respect to the overall charge neutrality point. The conductance trace in Fig. 2(d) mirrors the situation of the hole QD in Fig. 2(a). The $n$-type QD can also be charged one by one with individual electrons, proving the ambipolar operation of our bilayer QDs. The corresponding Coulomb diamonds for the electron dot [Fig. 2(e)] again mirror the situation of Fig. 2(c). In the electron as well as in the hole regime, charging energies on the order of $10 \mathrm{meV}$ are observed. In contrast to the $p$-type QD presented in Fig. 2(c), the $n$-type QD exhibits additional features in the region of zero charge carrier occupation of the QD. These features depend on the precise setting of the split gate voltage $V_{\mathrm{SG}}$ and correspond to localized states in the leads close to the QD (see Fig. 6). In total, eight different QDs were measured both in the electron and hole regime, all showing qualitatively the same results.

\section{B. Level structure}

In the second experiment at $1.7 \mathrm{~K}$, we measure Coulomb resonances of the electron QD 9 as a function of a perpendicular magnetic field $B_{\perp}$ [conductance map in Fig. 3(a)]. The shifts of the resonances in $V_{\mathrm{FG}}$ as a function of $B_{\perp}$ correspond to shifts of energy levels of the QD evolving with $B_{\perp}$. To extract the energy level spectrum of our QD from the resonance spacings, we subtract the charging energy $[21,35,36]$ by shifting neighboring resonances such that they touch in a single point and convert the voltage to an energy axis (see Appendix A). The extracted magnetic field dependence of the energy levels is shown in Fig. 3(b), where the color scale indicates the peak
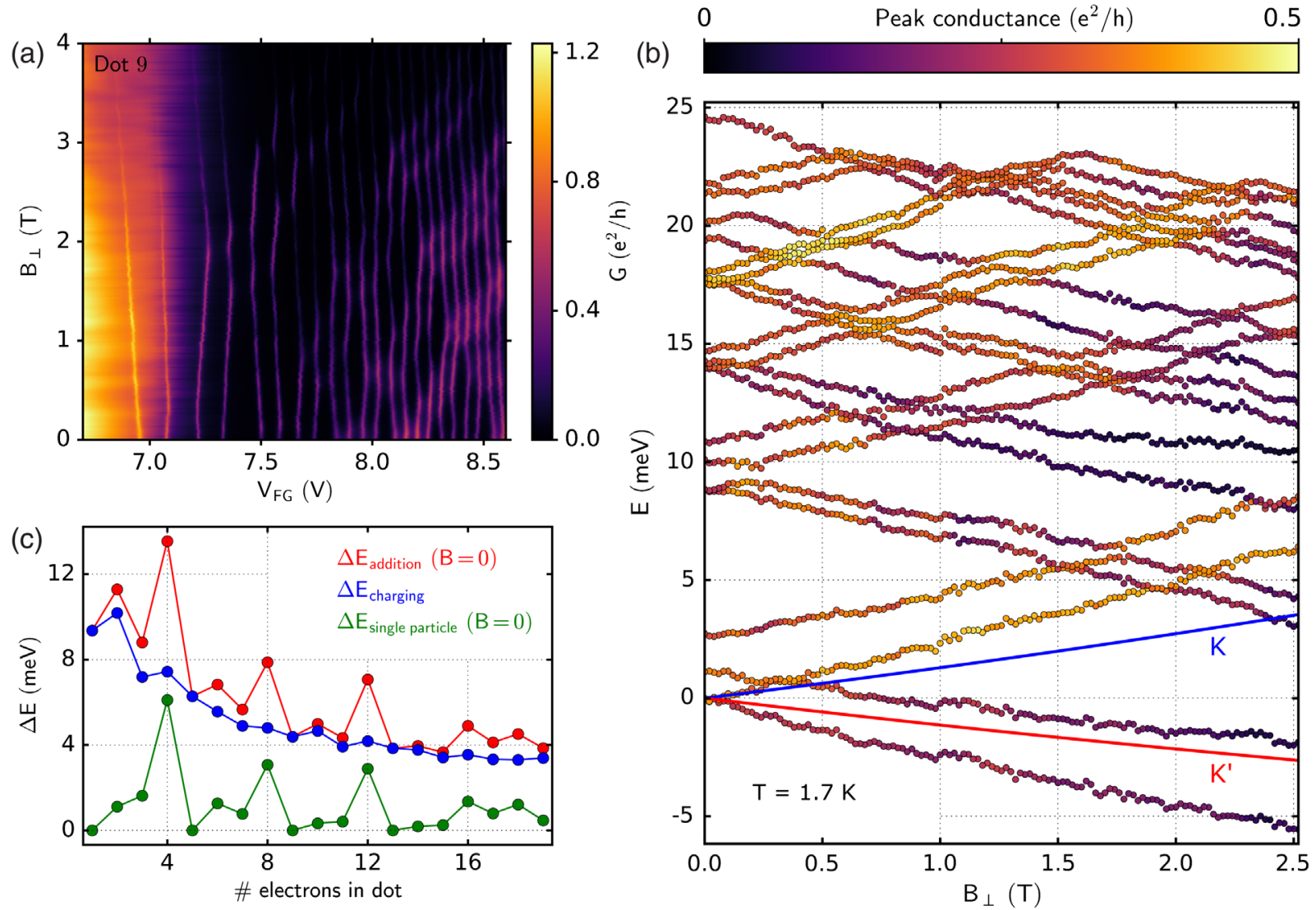

FIG. 3. (a) Conductance map in a perpendicular magnetic field for QD 9 in the electron regime. (b) Single particle energy level dispersion for QD 9 with perpendicular magnetic field $B_{\perp}$ extracted from Fig. 3(a). Blue and red solid lines are the result of theoretical calculations of the lowest energy levels. (c) Addition (red), charging (blue), and single particle energy (green) as a function of the QD occupation extracted from Fig. 3(a). 
conductance of each level, which is proportional to the coupling of the corresponding state to the leads [36].

We see that the levels bunch in groups of four at zero magnetic field, as expected from the twofold valley and twofold spin degeneracy in bilayer graphene, similar to carbon nanotubes $[24,26]$. Each shell of four states splits into $2+2$ as the magnetic field is increased, one pair shifting up, and the other down in energy. The splitting between these states is linear in the accessible magnetic field range, and is 40 times stronger than the Zeeman slitting for a free electron.

To compare the data with theory, we used the bilayer QD model presented in Ref. [14] and adapted it to our system. The allowed energy levels depend on the valley index (labeled by $\tau= \pm 1$ in the theory corresponding to the $K$ and $K^{\prime}$ valley), the angular momentum number $m$, the interlayer asymmetry $V$, the confinement potential $U$, and the radius $R$ of the QD. The levels have to be calculated numerically by matching four-component spinor states at the QD boundary. From the displacement field applied in the experiment, the interlayer asymmetry was estimated to be $V=60 \mathrm{meV}$ [37]. Since electrons are confined electrostatically, the confinement potential $U$ should be on the order of the interlayer asymmetry $V$ and we fix $U=V$. The remaining parameter $R$ determines both the orbital energy level difference at $B_{\perp}=0$ and the valley splitting as function of $B_{\perp}$. To reproduce the observed orbital energy level difference on the order of $5 \mathrm{meV}$, we obtain $R=20 \mathrm{~nm}$, which agrees well with the lithographic design of the device. The calculated energy levels (spin-degenerate in the theory) are shown in blue and red in Fig. 3(b) for the $\mathrm{K}$ and $\mathrm{K}$ ' states, respectively. To improve the theoretical model, it should be adapted to the nonradial symmetry of the dot and the nonhomogeneous confinement laterally and in the transport direction (see Appendix C). The experimentally observed valley splitting varies by $20 \%$ between different QDs, which could be a result of the microscopic differences in the size of the individual QDs caused in fabrication.

Figure 3(c) shows the experimental spacing of neighboring states as a function of occupation number. The single particle level spacing at zero field and the charging energy are shown in green and blue, respectively. The charging energy decreases with an increasing number of electrons occupying the QD, because the effective electronic dot becomes larger $[21,36]$. The addition energy, the sum of the two former, is directly proportional to the spacing of Coulomb resonances in finger gate voltage at zero field. We observe a clear fourfold level bunching [Figs. 3(b) and $3(\mathrm{c})]$, originating from the twofold spin and valley degeneracy of bilayer graphene [13-15], which can already be seen in Figs. 2(d) and 2(e). Until now, this intrinsic property specific to graphene QDs has not been observed experimentally. The same level bunching was also observed for the hole QDs at $35 \mathrm{mK}$ (see Appendix B).

\section{C. g-factor}

In the third experiment at $80 \mathrm{mK}$, we align the device parallel to the magnetic field in a revolving sample holder. The energy levels in Fig. 4(a) as a function of the parallel magnetic field are extracted in the same way as for Fig. 3(b), but vertically shifted by $1 \mathrm{meV}$ for clarity. Blue lines are guides to the eye for purely Zeeman split energy levels with a spin g-factor of $g_{s}=2$. Repeating the measurement of Fig. 4(a) for different rotation angles $\phi$ enables us to extract the spin Zeeman and orbital contribution to the splitting of energy levels as a function of the angle. The orbital contribution adds to the Zeeman splitting and is proportional to the perpendicular component of the magnetic field. With respect to horizontal lines in Fig. 4(a), the splitting is enhanced for levels $K^{\prime} \downarrow$ and $K \uparrow$ and is reduced for levels $K \downarrow$ and $K^{\prime} \uparrow$. Averaging over these pairs of levels leads to the data shown in Fig. 4(b), where solid lines represent fits to the data.

In theory, the red and blue points should touch at $g_{s}=2$ for perfect alignment of the sample parallel to the magnetic
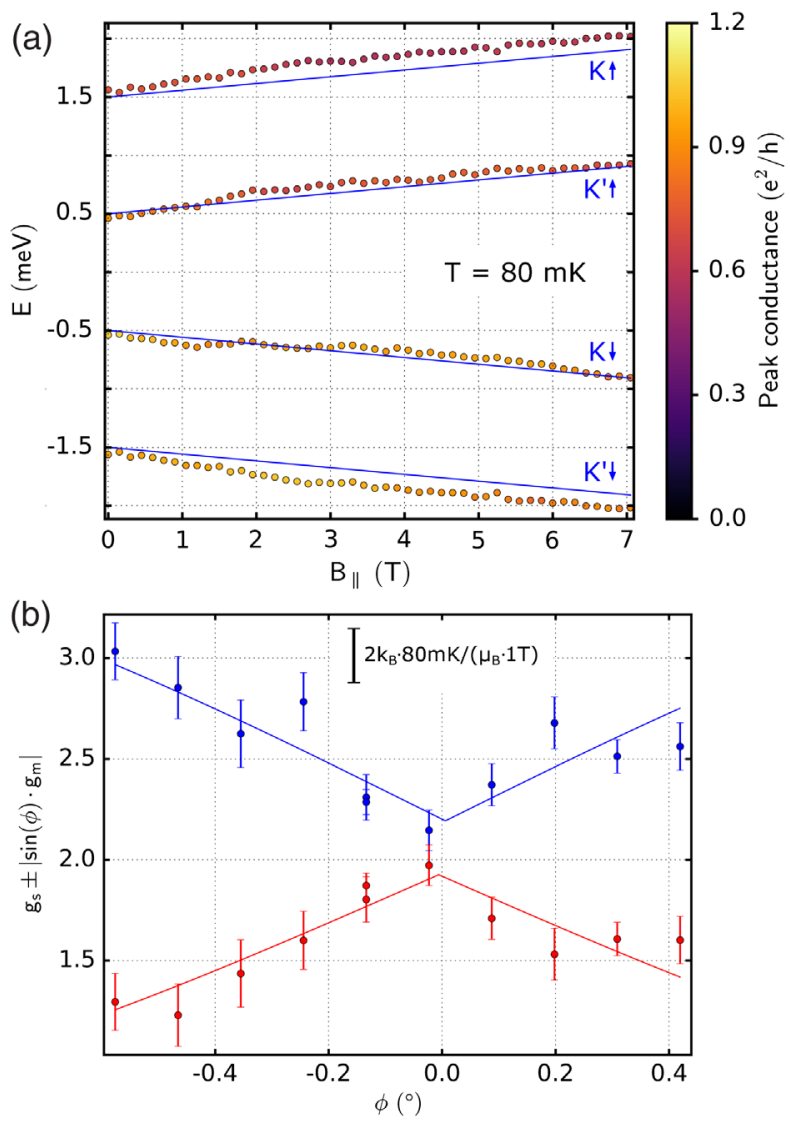

FIG. 4. (a) Lowest single particle energy levels of QD 2 in the electron regime as a function of parallel magnetic field. Energy levels are shifted by $1 \mathrm{meV}$ for clarity. (b) Sum of the spin Zeeman $\left(g_{s}\right)$ and orbital $\left(g_{m}\right)$ contribution to the energy level splitting as a function of the sample tilt angle $\phi$ with respect to the magnetic field. Blue (red) points are averaged over levels $K^{\prime} \downarrow$ and $K \uparrow$ (levels $K \downarrow$ and $K^{\prime} \uparrow$ ), and solid lines represent fits to the data. 
field, and the slope of the fits should correspond to the slopes of the lowest four levels in Fig. 3(b). The extracted orbital splitting for $\phi=90^{\circ}$ is 35 (blue) to 40 (red) times stronger than the Zeeman effect for a free electron, matching the experimental data from Fig. 3(b). The extracted spin g-factor of $g_{s}=2.08 \pm 0.22$ also agrees well with the predicted value for carbon-based devices [22,23].

With the experiments in parallel and perpendicular magnetic fields, we can show that the observed fourfold level bunching originates from the twofold spin degeneracy (split in $B_{\|}$) and the twofold valley degeneracy (split in $B_{\perp}$ ) of bilayer graphene.

Follow-up manuscripts reporting on double- and multiQD systems in bilayer graphene have, meanwhile, appeared in the literature $[38,39]$.

\section{CONCLUSION}

The presented experimental results and the qualitative agreement with theoretical calculations prove the quality and understanding of our bilayer QDs. The electrostatic confinement of single charge carriers in a planar technology is an important step toward the promising implementation of spin and valley qubits in graphene-based devices. With the $p-n$ junctions serving as natural tunnel barriers, QDs can be coupled in the future to create a series of QDs with alternating polarity. We expect that the implementation of highfrequency read-out will enable the determination of spin and valley coherence in graphene quantum dots and open up new horizons for spin and valley qubit research.

\section{ACKNOWLEDGMENTS}

We thank Jelena Klinovaja and Francois Peeters for fruitful discussions, and Peter Märki, Erwin Studer, as well as the FIRST staff for their technical support. We also acknowledge financial support from the European Graphene Flagship, the Swiss National Science Foundation via NCCR Quantum Science and Technology, the EU Spin-Nano RTN network and ETH Zurich via the ETH fellowship program. Growth of hexagonal boron nitride crystals was supported by the Elemental Strategy Initiative conducted by the MEXT, Japan, and JSPS KAKENHI Grant No. JP15K21722.

Author contributions: M.E. and R. P. fabricated the device. M.E. performed the measurements. F.H. performed the theoretical calculations. H. O., A. K., Y.L., and P. R. supported device fabrication and data analysis. $\mathrm{K}$. W. and T. T. provided high-quality boron nitride crystals. K. E., T. I., and M. S. supervised the work. The authors declare that they have no competing interests.

\section{APPENDIX A: MATERIALS AND METHODS}

\section{Fabrication}

The device was fabricated as described in Ref. [19]. The van der Waals heterostructure was built up using the pick-up technique described in Ref. [33]. The stacked heterostructure was then deposited on a phosphorus-doped $\mathrm{Si}$ chip with a dielectric layer of $285 \mathrm{~nm} \mathrm{SiO}$. From the bottom to the top [see Fig. 1(b)], the stack contains a thin graphite flake (graphite back gate), the approximately 27-nm-thick bottom boron nitride flake, the approximately $20-\mu \mathrm{m}$-long and $1-\mu \mathrm{m}$-wide bilayer flake, and the approximately $24-\mathrm{nm}$-thick top boron nitride flake. After encapsulating the bilayer graphene in boron nitride [32], the bilayer flake is protected from the following processing steps. We work with the natural shape of the exfoliated bilayer flake to protect its natural edges. In a first processing step, side contacts [33] were patterned using electron-beam lithography (EBL) and etched by reactiveion etching (40 sccm of $\mathrm{CHF}_{3}, 4 \mathrm{sccm}$ of $\mathrm{O}_{2}, 132 \mathrm{~W} \mathrm{RF}$ power, $306 \mathrm{~V}$ DC bias), followed by evaporation of $10 \mathrm{~nm}$ chromium and $60 \mathrm{~nm}$ gold. The two top gate layers [green and blue gates in Fig. 1(a)] are both produced in two consecutive EBL steps. The inner gate structure on top of the bilayer flake is written in a separate EBL step to achieve high resolution with a thin EBL resist. The thinner resist allows only for evaporating $5 \mathrm{~nm}$ chromium and $25 \mathrm{~nm}$ gold. In a second step with thicker EBL resist, the outer gate structure connecting to the bond pads is written and $10 \mathrm{~nm}$ chromium and $60 \mathrm{~nm}$ gold are evaporated. For the first top gate layer, an additional gentle reactive-ion etching step (80 sccm of Ar, $5 \mathrm{sccm}$ of $\mathrm{O}_{2}, 70 \mathrm{~W}$ RF power, $222 \mathrm{~V}$ DC bias) is performed to ensure that the gates are sticking on the boron nitride surface. To separate the two top gate layers, we grow an approximately 25-nm-thick layer of $\mathrm{Al}_{2} \mathrm{O}_{3}$ by atomic layer deposition at $150^{\circ} \mathrm{C}$ (with precursor gases of trimethylaluminum and water).

The device was cooled down four times in different setups: a dipstick ${ }^{4} \mathrm{He}$ system at $4.2 \mathrm{~K}$, a variable temperature insert reaching $1.7 \mathrm{~K}$, a dry dilution refrigerator reaching $35 \mathrm{mK}$, and a wet dilution refrigerator with a rotatable sample holder reaching $80 \mathrm{mK}$.

\section{Extracting energy levels}

Figure 3(a) shows Coulomb resonances as a function of $B_{\perp}$. The finger gate axis can be converted into an energy axis, using the Coulomb diamond measurement and determining the lever arm $\alpha$ of the finger gate [36]. To add an additional electron to the QD, the electron has to pay charging energy (due to Coulomb interaction) in addition to the single particle energy level difference. Thus, when two single particle levels of the QD are degenerate in energy, the additional electron only pays charging energy, which is assumed to be independent of the magnetic field. In turn, this means that the minimal distance of Coulomb resonances in $\alpha V_{\mathrm{FG}}$ corresponds exactly to the charging energy. Subtracting the charging energy means shifting neighboring resonances in $\alpha V_{\mathrm{FG}}$ such that they touch at one point. The result is shown in Fig. 3(b), yielding the single particle energy level spectrum of the QD. Figure 3(c) shows the 
subtracted charging energy (blue curve), which decreases as expected with increasing electron occupation number [21]. The single particle energy (green curve) shows clear peaks whenever the occupation number is an integer multiple of 4. Therefore, also, the addition energy (red curve) shows maxima at the same position, which is why the fourfold level bunching can already be observed in the Coulomb resonances of the conductance traces (see Fig. 6).

\section{APPENDIX B: SUPPORTING DATA}

\section{Dirac points}

In order to demonstrate the high electronic quality of our sample, Fig. 5(a) shows a resistance map as a function of graphite back gate voltage $V_{\mathrm{BG}}$ and top gate voltage $V_{\mathrm{TG}}$ applied to a uniform top gate crossing the entire bilayer region [gate marked by a white asterisk in Fig. 1(a)]. All other gates (split and finger gates) are grounded for this measurement.

The overall Dirac point [white triangle in Fig. 5(a)] is reached when $V_{\mathrm{BG}}=-0.34 \mathrm{~V}$ and $V_{\mathrm{TG}}=-0.62 \mathrm{~V}$. The horizontal line of high resistance at $V_{\mathrm{BG}}=-0.34 \mathrm{~V}$ [white diamond in Fig. 5(a)] corresponds to the Dirac point of the bilayer regions above the graphite back gate [red solid line in Fig. 1(a)], not being covered by any top gates [green gates in Fig. 1(a)]. The second horizontal line of high resistance [white square in Fig. 5(a)] corresponds to the Dirac peak underneath the two pairs of split gates kept at $V_{\mathrm{SG}}=0$. To induce the same density in these regions compared to the regions not covered by any gates on top, the split gates would have to be set to $V_{\mathrm{SG}}=-0.62 \mathrm{~V}$. With the slope of the diagonal resistance peak, we can calculate the offset in $V_{\mathrm{BG}}$ that would compensate for the offset in $V_{\mathrm{SG}}$. We find $\Delta V_{\mathrm{BG}}=0.95 \mathrm{~V}$, which agrees with the distance of the two horizontal resistance peaks in $V_{\mathrm{BG}}$. To recapitulate, the high resistance along the two horizontal lines corresponds to the Dirac points in the regions above the graphite back gate covered and not covered by grounded split gates.

\section{Pinch-off resistance}

The high resistance along the $D$-axis in Fig. 5(a) corresponds to the Dirac peak underneath the biased top gate [white asterisk in Fig. 1(a)], spanning the whole width of the bilayer flake. Along this line, away from the overall Dirac point [white triangle in Fig. 5(a)], the displacement field in the bilayer increases, leading to the opening of a band gap in the region below the top gate [10]. At high displacement field [red circle in Fig. 5(a)], resistances in the $\mathrm{G} \Omega$ regime can be reached [see Fig. 5(b)]. This is the regime in which we operate our QDs.

The measurement shown in Fig. 5(a) was performed with a constant source-drain bias excitation of $500 \mu \mathrm{V}$. We measure the current with a precision of $10 \mathrm{pA}$ using an IV converter. This means that we can only measure resistances
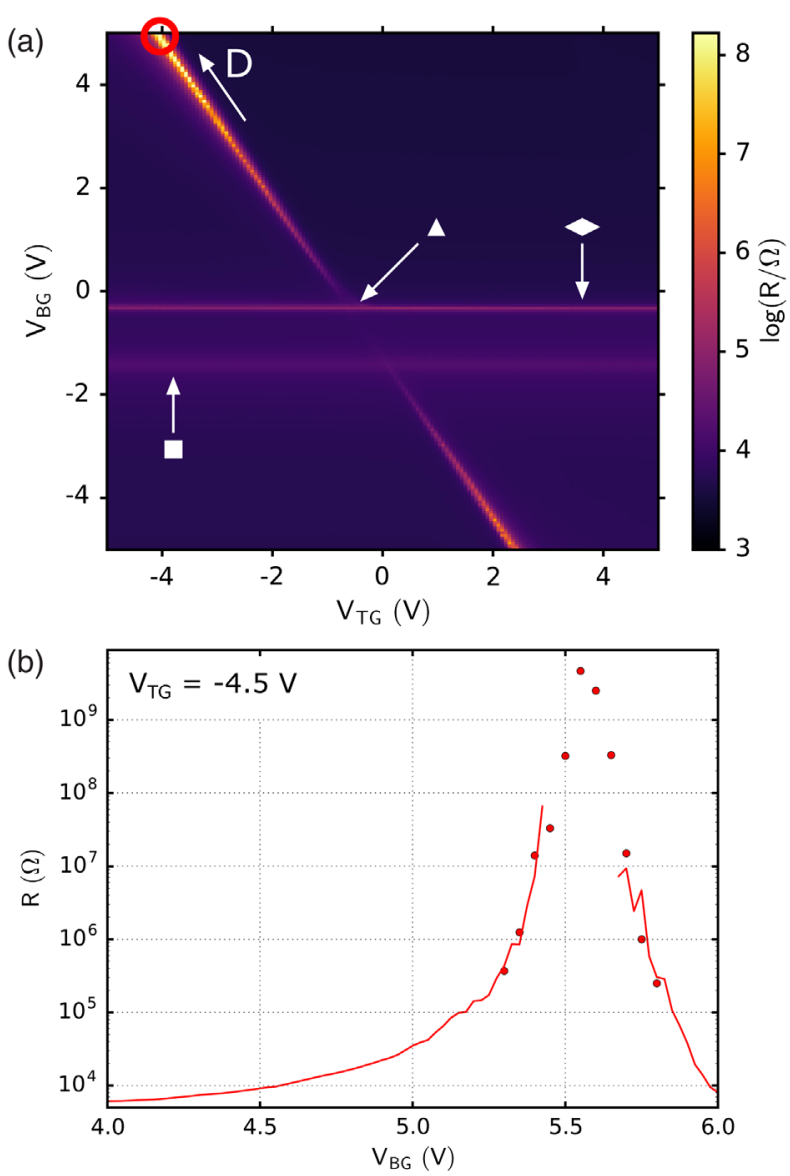

FIG. 5. (a) Logarithmic two-terminal resistance of the device as a function of the graphite back gate voltage $V_{\mathrm{BG}}$ and the top gate voltage $V_{\mathrm{TG}}$ [asterisk in Fig. 1(a)]. (b) Two-terminal resistance as a function of the graphite back gate voltage at fixed top gate voltage $V_{\mathrm{TG}}=-4.5 \mathrm{~V}$. The solid line was measured in a constant source-drain bias setup, while the circles correspond to resistance values extracted from IV characteristics recorded at the given gate voltage setting.

up to $50 \mathrm{M} \Omega$. The extracted resistance values as a function of $V_{\mathrm{BG}}$ at $V_{\mathrm{TG}}=-4.5 \mathrm{~V}$ are plotted as a solid line in Fig. 5(b). When the Fermi energy lies within the gap opened by the strong displacement field, the resistance reaches higher values and the measured current approaches zero. Therefore, we record additional IV characteristics of the device in this regime. The extracted resistance values are plotted as solid circles in Fig. 5(b), reaching resistances in the $\mathrm{G} \Omega$ regime.

\section{Tuning into the $\mathrm{QD}$ regime}

The Coulomb resonances (white star in Fig. 6) of QD1 in the hole regime can be clearly identified in the logarithmic conductance map as a function of channel and split gate voltage. The middle region of low conductance corresponds to charge neutrality underneath the split gates such that transport outside of the channel is strongly suppressed. At more negative (positive) split gate voltage, $p$-type ( $n$-type) 


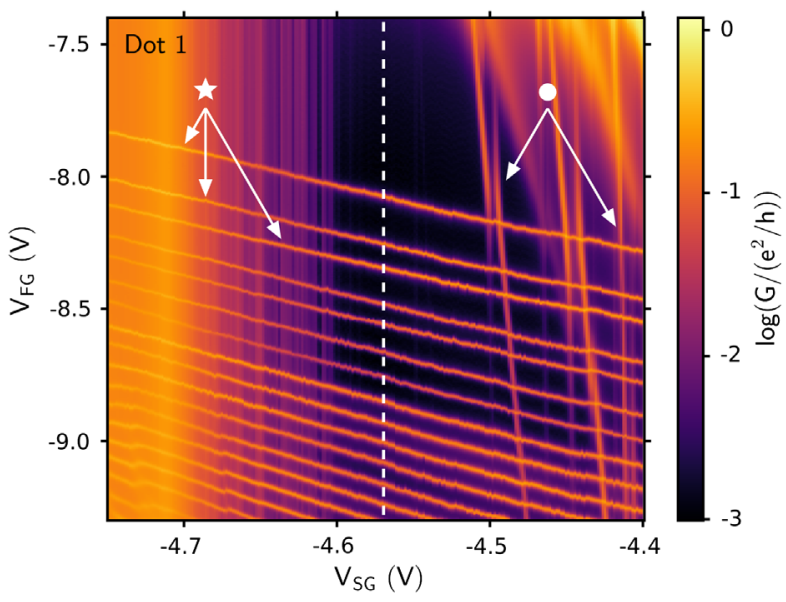

FIG. 6. Logarithmic two-terminal conductance as a function of the split gate voltage $V_{\mathrm{SG}}$ and the finger gate voltage $V_{\mathrm{FG}}$ for QD 1 measured at $V_{\mathrm{BG}}=5.55 \mathrm{~V}$. The cut along the dashed line corresponds to Fig. 2(a).
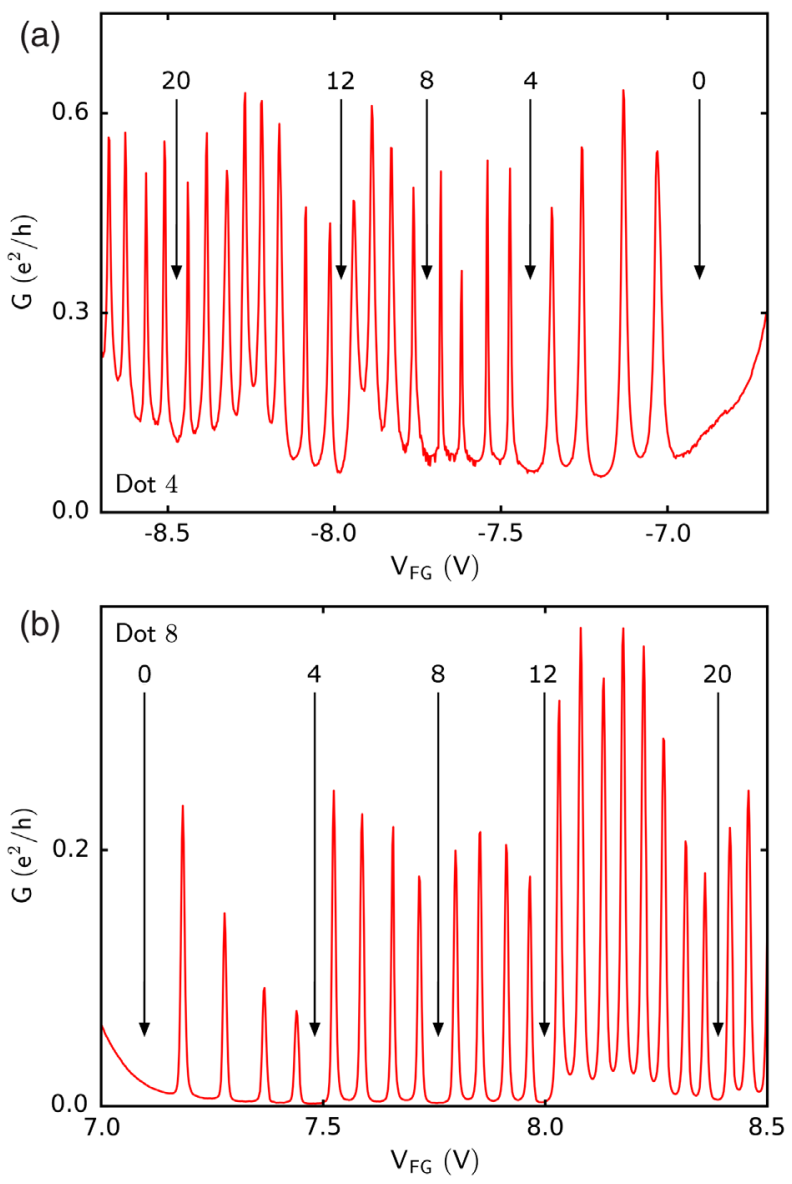

FIG. 7. Conductance traces for (a) QD 4 in the hole regime and (b) QD 8 in the electron regime measured at $35 \mathrm{mK}$ and $1.7 \mathrm{~K}$, respectively. Numbers indicate the occupation number of the respective QD. conductance underneath the split gates leads to increased conductance parallel to the channel. In addition to the Coulomb resonances, localized states close to the QD can be identified (white circle in Fig. 6). However, they do not influence the QD at the operation point indicated by the dashed line [cut along the dashed line corresponds to Fig. 2(a)].

The cross capacitance of the QD and the split gates leads to the finite slope of the Coulomb resonances in Fig. 6. Additionally, increasing the voltage $V_{\mathrm{SG}}$ applied to all split gates squeezes the hole wave function of the $\mathrm{QD}$, leading to an increase in confinement energy and, thus, Coulomb resonance spacing.

\section{Level bunching}

As mentioned in the main text of the manuscript, the hole QDs also showed a fourfold level bunching in the cooldown to $35 \mathrm{mK}$. The corresponding conductance curve as a function of the finger gate voltage for QD 8 in the hole regime is shown in Fig. 7(a). Numbers indicate the occupation of the QD with holes, and we observe an increased addition energy whenever the occupation number is an integer multiple of 4 (with the exception of 16). This agrees with shell filling of our QDs [36] and the fourfold degeneracy of bilayer graphene.

Figure 7(b) shows the same measurement for QD 4 in the electron regime, mirroring the result of Fig. 7(a). Overall, 8 of our 11 QDs show qualitatively the same behavior, both in the electron and in the hole regime. All QDs show a sequence of groups of 4 levels, sometimes with groups of 8 or 12 showing up, depending on the specific QD.

\section{APPENDIX C: THEORETICAL CONSIDERATIONS}

For comparison of the measured data with theory, we adopted the solution for the energy levels of a bilayer QD in a perpendicular magnetic field [14]. The allowed energy levels depend on the valley number $\tau$ ( +1 for $K$ and -1 for $K^{\prime}$ ), the total angular momentum $m$, the interlayer potential asymmetry $V$ (potential difference between upper and lower layer), the boundary potential $U$, and the radius $R$ of the circular dot. The resulting energy levels are determined numerically.

\section{Ground state}

The result of fitting the ground state is shown in Fig. 3(b). The fit was performed with fixed $U=$ $V=60 \mathrm{meV}$, calculated from the voltages applied in the experiment. We find $R=20 \mathrm{~nm}$, where the ground state has the total angular momentum number $m=+1$. We observe that the parameters $U$ and $R$ have the strongest influence on the orbital energy level spacing (approximately $5-10 \mathrm{meV}$ in the experiment) and their magnetic field dependence for small magnetic fields $\left(B_{\perp} \sim 0-2.5 \mathrm{~T}\right)$. 
TABLE I. Matrix element $M$ introduced in Eq. (C2), calculated for the first four lowest energy levels of the bilayer quantum dot model presented in Ref. [14], considering $U=V=60 \mathrm{meV}$ and $R=20 \mathrm{~nm}$.

\begin{tabular}{ccccc}
\hline \hline$m$ & 1 & 0 & 2 & -1 \\
\hline$M$ & 0.17 & 0.18 & 0.33 & 0.06 \\
\hline \hline
\end{tabular}

\section{Linear magnetic field regime}

Since the experimental results in Fig. 3(b) show an essentially linear magnetic-field dependence of the energy levels in the covered field range, we use a perturbative approach starting from the zero-field lowest energy states. We analyze the linear correction to the energy of the states in lowest-order perturbation theory in $B_{\perp}$ and obtain an estimate for the orbital g-factor $g_{\text {orb }}$. Based on the theoretical approach in Ref. [14], we write the eigenstates as

$$
\Psi(\rho, \phi)=\frac{e^{i m \phi}}{\sqrt{\rho}}\left(\begin{array}{cccc}
1 & 0 & 0 & 0 \\
0 & e^{-i \phi} & 0 & 0 \\
0 & 0 & 1 & 0 \\
0 & 0 & 0 & e^{+i \phi}
\end{array}\right) \psi_{m}(\rho)
$$

where $\psi_{m}(\rho)$ is the radial part of the Dirac spinor depending on $m$ and the radius $\rho=r / R$. We then use the solution of the ground state to calculate the correction to the energy level due to the magnetic field in first-order perturbation as

$$
\begin{aligned}
E_{B} & =\tau \frac{R}{l_{F}} \underbrace{\left|\frac{\int_{0}^{\infty} d \rho \rho \psi_{m}(\rho)^{\dagger} \gamma^{5} \psi_{m}(\rho)}{\int_{0}^{\infty} d \rho \psi_{m}(\rho)^{\dagger} \psi_{m}(\rho)}\right|}_{M} \mu_{B} B_{\perp} \\
& =g_{\mathrm{orb}} \mu_{B} B_{\perp},
\end{aligned}
$$

where we introduced the Fermi length $l_{F}=\hbar /\left(v_{F} m_{e}\right)$, which has, considering $v_{F}=10^{6} \mathrm{~m} / \mathrm{s}$, the value
$l_{F}=1.16 \AA$. The $4 \times 4$ matrix $\gamma^{5}$ can be expressed by the Pauli matrices as

$$
\gamma^{5}=\left(\begin{array}{cc}
\sigma^{2} & 0 \\
0 & -\sigma^{2}
\end{array}\right)
$$

Note that the transformation $(m, \tau) \rightarrow(-m,-\tau)$ simply leads to a change of sign in $E_{B}$.

Let us elaborate briefly on this approximation of $E_{B}$. The perturbative result only applies in the field range where the correction to the zero-field energy is smaller than the energy level spacing at $B_{\perp}=0$. Through the spinor $\psi_{m}(\rho)$, $g_{\text {orb }}$ depends on both $m$ and $U$. However, the matrix element $M$ in Eq. (C2) varies only between 0.05 and 0.35 , as can be seen in Table I, such that the strongest effect on $g_{\text {orb }}$ actually originates from the ratio $R / l_{F}$. The difference between the exact and the perturbative result is small in the low-field limit.

\section{Noncircular dots}

We briefly want to discuss the effect of a modified boundary potential $U$, for which we have so far taken a hard wall potential, $U(x, y)=0$ for $r \leq R$ and $U$ for $r>R$ $\left(r^{2}=x^{2}+y^{2}\right)$. We study the effect of a more general potential, which may be noncircular and layer dependent.

This modification is responsible for two effects. The layer dependence of the potential lifts the degeneracy at $B_{\perp}=0$. In addition, the noncircular structure yields an energy shift, which again can be calculated by means of perturbation theory to lowest order,

$$
E_{U}=\frac{\int d x d y \Psi(x, y)^{\dagger} U(x, y) \Psi(x, y)}{\int d x d y \Psi(x, y)^{\dagger} \Psi(x, y)} .
$$

In order to examine the effect of $B_{\perp}$ on this shift, we consider the shape of the radial wave function, $|\Psi(\rho)|^{2}=$ $\psi_{m}(\rho)^{\dagger} \psi_{m}(\rho)$ (including both valleys, $\tau= \pm 1$ ), plotted in Fig. 8 for fields $B_{\perp}=0,2.5 \mathrm{~T}$. In the legend, the states are

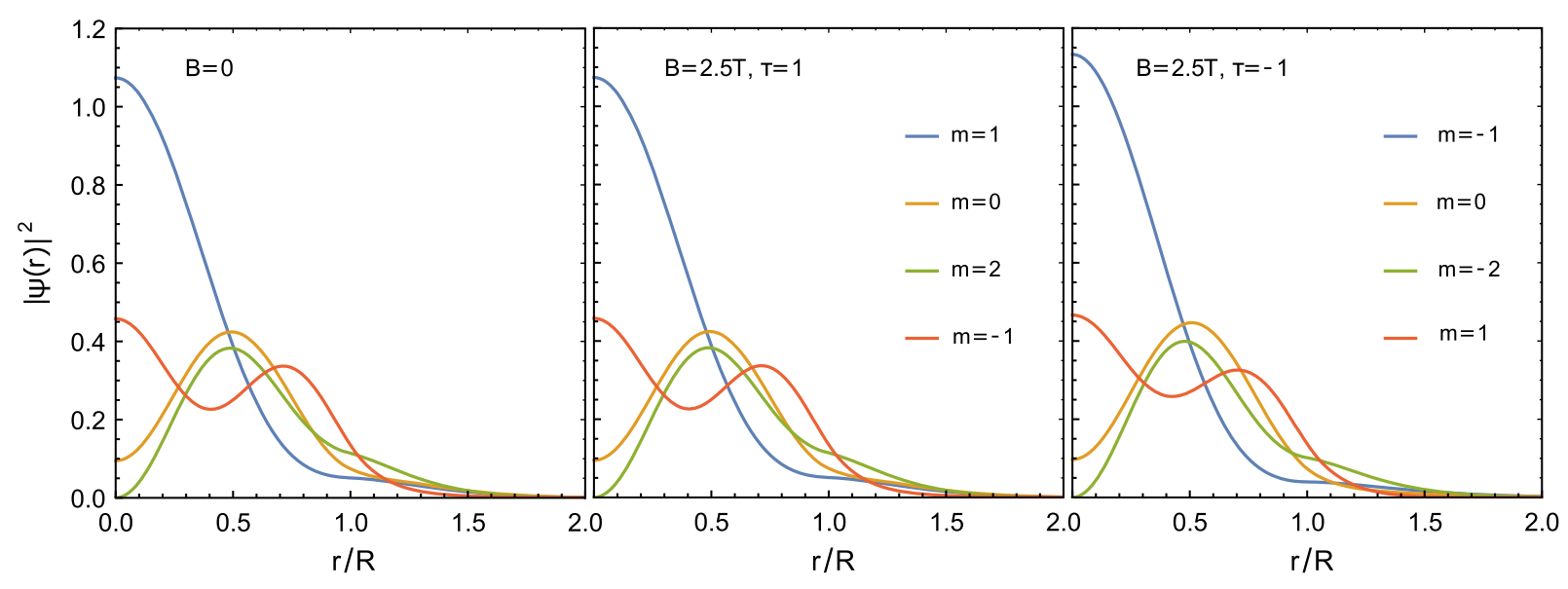

FIG. 8. $|\Psi(r)|^{2}$ for the four lowest energy levels in both valleys considering $R=20 \mathrm{~nm}$ and $U=V=60 \mathrm{meV}$. 


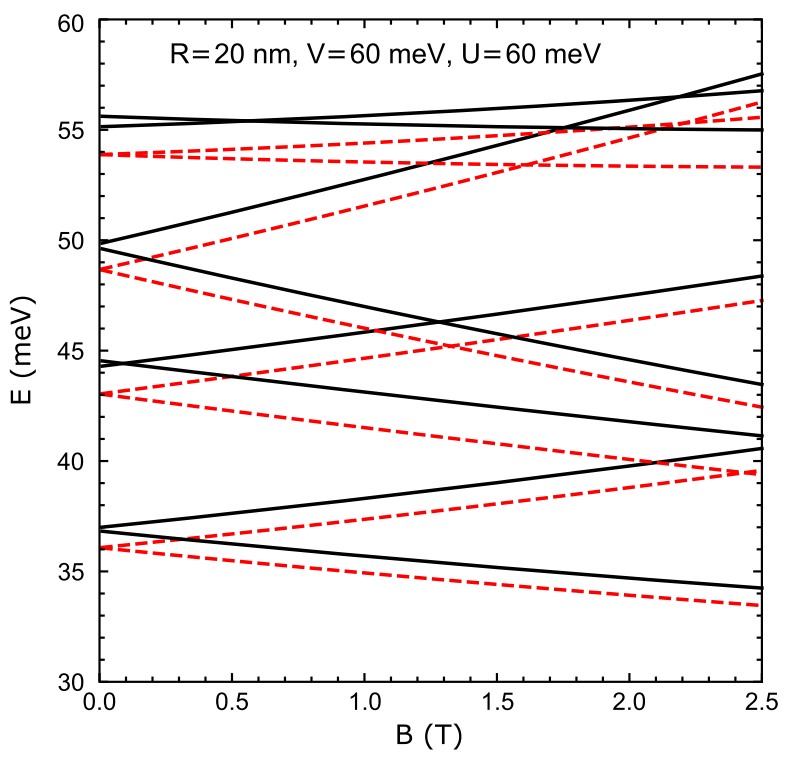

FIG. 9. Zeroth-order (red) and first-order (black) energy levels for the described model with the boundary potential $U(x, y)$ (the real boundary will be some complicated function of $r$ ).

ordered according to their energy, with $m=1$ being the ground state. It becomes obvious that the magnetic field only slightly influences $|\Psi(\rho)|^{2}$ for all displayed $m$. Therefore, it is clear that the energy shift due to the modified boundary potential leads to an essentially fieldindependent shift of the energy, i.e., $g_{\text {orb }}$ remains practically unchanged, if the mean radius $R$ is kept constant.

To demonstrate this effect, we use $U(x, y)=U_{L}\left[\left(x^{2} / R^{2}\right)+\right.$ $\left.\chi_{L}\left(y^{2} / R^{2}\right)\right]$ for $x^{2}+\chi_{L} y^{2} \leq R^{2}$ and $U(x, y)=U$ for $x^{2}+\chi_{L} y^{2} \geq R^{2}$, where $L=1,2$ is a layer index and $\chi_{L}$ a generally layer-dependent anisotropy parameter. In Fig. 9 , we compare the result of the standard hard wall potential (red dashed) with the layer-dependent potential above (black solid) using the parameters $U_{1}=U_{2}=6 \mathrm{meV}, \chi_{1}=0.9$, and $\chi_{2}=0.95$, while keeping $U=60 \mathrm{meV}$.

The small potential difference between the two layers leads to a splitting of the degeneracy of the pair $(m, \tau)$ and $(-m,-\tau)$ at $B_{\perp}=0$, which might be one of the reasons for the observed splitting of energy levels at $B_{\perp}=0$ in the experiment.

[1] B. Trauzettel, D. V. Bulaev, D. Loss, and G. Burkard, Spin Qubits in Graphene Quantum Dots, Nat. Phys. 3, 192 (2007).

[2] C. Stampfer, E. Schurtenberger, F. Molitor, J. Güttinger, T. Ihn, and K. Ensslin, Tunable Graphene Single Electron Transistor, Nano Lett. 8, 2378 (2008).

[3] L. A. Ponomarenko, F. Schedin, M. I. Katsnelson, R. Yang, E. W. Hill, K. S. Novoselov, and A. K. Geim, Chaotic Dirac Billiard in Graphene Quantum Dots, Science 320, 356 (2008).
[4] X. L. Liu, D. Hug, and L. M. K. Vandersypen, Gate-Defined Graphene Double Quantum Dot and Excited State Spectroscopy, Nano Lett. 10, 1623 (2010).

[5] J. Güttinger, F. Molitor, C. Stampfer, S. Schnez, A. Jacobsen, S. Dröscher, T. Ihn, and K. Ensslin, Transport through Graphene Quantum Dots, Rep. Prog. Phys. 75, 126502 (2012).

[6] D. Bischoff, A. Varlet, P. Simonet, M. Eich, H. C. Overweg, T. Ihn, and K. Ensslin, Localized Charge Carriers in Graphene Nanodevices, Appl. Phys. Rev. 2, 031301 (2015).

[7] J. Güttinger, C. Stampfer, S. Hellmüller, F. Molitor, T. Ihn, and K. Ensslin, Charge Detection in Graphene Quantum Dots, Appl. Phys. Lett. 93, 212102 (2008).

[8] F. Miao, S. Wijeratne, Y. Zhang, U. C. Coskun, W. Bao, and C. N. Lau, Phase-Coherent Transport in Graphene Quantum Billiards, Science 317, 1530 (2007).

[9] S. Russo, J. B. Oostinga, D. Wehenkel, H. B. Heersche, S. S. Sobhani, L. M. K. Vandersypen, and A. F. Morpurgo, Observation of Aharonov-Bohm Conductance Oscillations in a Graphene Ring, Phys. Rev. B 77, 085413 (2008).

[10] E. McCann, Asymmetry Gap in the Electronic Band Structure of Bilayer Graphene, Phys. Rev. B 74, 161403 (2006).

[11] T. Ohta, A. Bostwick, T. Seyller, K. Horn, and E. Rotenberg, Controlling the Electronic Structure of Bilayer Graphene, Science 313, 951 (2006).

[12] J. B. Oostinga, H. B. Heersche, X. Liu, A. F. Morpurgo, and L. M. K. Vandersypen, Gate-Induced Insulating State in Bilayer Graphene Devices, Nat. Mater. 7, 151 (2008).

[13] J. M. Pereira, P. Vasilopoulos, and F. M. Peeters, Tunable Quantum Dots in Bilayer Graphene, Nano Lett. 7, 946 (2007).

[14] P. Recher, J. Nilsson, G. Burkard, and B. Trauzettel, Bound States and Magnetic Field Induced Valley Splitting in GateTunable Graphene Quantum Dots, Phys. Rev. B 79, 085407 (2009).

[15] M. Zarenia, B. Partoens, T. Chakraborty, and F. M. Peeters, Electron-Electron Interactions in Bilayer Graphene Quantum Dots, Phys. Rev. B 88, 245432 (2013).

[16] M. T. Allen, J. Martin, and A. Yacoby, Gate-Defined Quantum Confinement in Suspended Bilayer Graphene, Nat. Commun. 3, 934 (2012).

[17] A. M. Goossens, S. C. M. Driessen, T. A. Baart, K. Watanabe, T. Taniguchi, and L. M. K. Vandersypen, GateDefined Confinement in Bilayer Graphene-Hexagonal Boron Nitride Hybrid Devices, Nano Lett. 12, 4656 (2012).

[18] M. J. Zhu, A. V. Kretinin, M. D. Thompson, D. A. Bandurin, S. Hu, G. L. Yu, J. Birkbeck, A. Mishchenko, I. J. VeraMarun, K. Watanabe, T. Taniguchi, M. Polini, J. R. Prance, K. S. Novoselov, A. K. Geim, and M. Ben Shalom, Edge Currents Shunt the Insulating Bulk in Gapped Graphene, Nat. Commun. 8, 14552 (2017).

[19] H. Overweg, H. Eggimann, X. Chen, S. Slizovskiy, M. Eich, R. Pisoni, Y. Lee, P. Rickhaus, K. Watanabe, T. Taniguchi, V. Fal'ko, T. Ihn, and K. Ensslin, Electrostatically Induced Quantum Point Contact in Bilayer Graphene, Nano Lett. 18, 553 (2018).

[20] F. A. Zwanenburg, A. S. Dzurak, A. Morello, M. Y. Simmons, L. C. L. Hollenberg, G. Klimeck, S. Rogge, 
S. N. Coppersmith, and M. A. Eriksson, Silicon Quantum Electronics, Rev. Mod. Phys. 85, 961 (2013).

[21] L. P. Kouwenhoven, D. G. Austing, and S. Tarucha, FewElectron Quantum Dots, Rep. Prog. Phys. 64, 701 (2001).

[22] A. Thess, R. Lee, P. Nikolaev, H. Dai, P. Petit, J. Robert, C. Xu, Y. H. Lee, S. G. Kim, A. G. Rinzler, D. T. Colbert, G. E. Scuseria, D. Tomnek, J.E. Fischer, and R. E. Smalley, Crystalline Ropes of Metallic Carbon Nanotubes, Science 273, 483 (1996).

[23] S. J. Tans, M. H. Devoret, H. Dai, A. Thess, R. E. Smalley, L. J. Geerligs, and C. Dekker, Individual Single-Wall Carbon Nanotubes as Quantum Wires, Nature (London) 386, 474 (1997).

[24] D. H. Cobden and J. Nygård, Shell Filling in Closed SingleWall Carbon Nanotube Quantum Dots, Phys. Rev. Lett. 89, 046803 (2002).

[25] P. Jarillo-Herrero, S. Sapmaz, C. Dekker, L. P. Kouwenhoven, and H.S. J. van der Zant, Electron-Hole Symmetry in a Semiconducting Carbon Nanotube Quantum Dot, Nature (London) 429, 389 (2004).

[26] S. Sapmaz, P. Jarillo-Herrero, L. P. Kouwenhoven, and H. S. J. van der Zant, Quantum Dots in Carbon Nanotubes, Semicond. Sci. Technol. 21, S52 (2006).

[27] G. A. Steele, F. Pei, E. A. Laird, J. M. Jol, H. B. Meerwaldt, and L. P. Kouwenhoven, Large Spin-Orbit Coupling in Carbon Nanotubes, Nat. Commun. 4, 1573 (2013).

[28] E. A. Laird, F. Pei, and L. P. Kouwenhoven, A Valley-Spin Qubit in a Carbon Nanotube, Nat. Nanotechnol. 8, 565 (2013).

[29] A. K. Geim and I. V. Grigorieva, Van der Waals Heterostructures, Nature (London) 499, 419 (2013).

[30] K. S. Novoselov, A. Mishchenko, A. Carvalho, and A. H. Castro Neto, $2 d$ Materials and van der Waals Heterostructures, Science 353, aac9439 (2016).

[31] S. J. McDonnell and R. M. Wallace, Atomically-Thin Layered Films for Device Applications Based upon $2 d$ TMDC Materials, Thin Solid Films 616, 482 (2016).
[32] C. R. Dean, A. F. Young, I. Meric, C. Lee, L. Wang, S. Sorgenfrei, K. Watanabe, T. Taniguchi, P. Kim, K. L. Shepard, and J. Hone, Boron Nitride Substrates for HighQuality Graphene Electronics, Nat. Nanotechnol. 5, 722 (2010).

[33] L. Wang, I. Meric, P. Y. Huang, Q. Gao, Y. Gao, H. Tran, T. Taniguchi, K. Watanabe, L. M. Campos, D. A. Muller, J. Guo, P. Kim, J. Hone, K. L. Shepard, and C. R. Dean, OneDimensional Electrical Contact to a Two-Dimensional Material, Science 342, 614 (2013).

[34] T. Uwanno, Y. Hattori, T. Taniguchi, K. Watanabe, and K. Nagashio, Fully Dry PMMA Transfer of Graphene on h-BN Using a Heating/Cooling System, 2D Mater. 2, 041002 (2015).

[35] A. Fuhrer, S. Lüscher, T. Ihn, T. Heinzel, K. Ensslin, W. Wegscheider, and M. Bichler, Energy Spectra of Quantum Rings, Nature (London) 413, 822 (2001).

[36] T. Ihn, Semiconductor Nanostructures: Quantum States and Electronic Transport (Oxford University Press, Oxford, New York, 2009).

[37] E. McCann and M. Koshino, The Electronic Properties of Bilayer Graphene, Rep. Prog. Phys. 76, 056503 (2013).

[38] M. Eich, R. Pisoni, A. Pally, H. Overweg, A. Kurzmann, Y. Lee, P. Rickhaus, K. Watanabe, T. Taniguchi, K. Ensslin, and T. Ihn, Coupled Quantum Dots in Bilayer Graphene, Nano Lett., DOI: 10.1021/acs.nanolett.8b01859 (2018).

[39] L. Banszerus, B. Frohn, A. Epping, D. Neumaier, K. Watanabe, T. Taniguchi, and C. Stampfer, Gate-Defined Electron-Hole Double Dots in Bilayer Graphene, Nano Lett., DOI: 10.1021/acs.nanolett.8b01303 (2018).

Correction: The author order was presented incorrectly and has been fixed. 